\title{
Consumo lácteo y su impacto en la salud ósea de población adulta en Colombia. Recomendaciones de la Asociación Colombiana de Osteoporosis y Metabolismo Mineral, basadas en la evidencia
}

\author{
Milk consumption and its impact on bone health of the adult population \\ in Colombia. Evidence-based recommendations of Asociación \\ Colombiana de Osteoporosis y Metabolismo Mineral
}

Medina $A,{ }^{1}$ Rosero $O,{ }^{2}$ Márquez $C L,{ }^{3}$ Coy $A F,{ }^{4}$ Ely $A L,{ }^{5}$ Rivera $A,{ }^{6}$ Benavidez $J,{ }^{7}$ Vargas $Y,{ }^{8}$ Obregón $M C,{ }^{9}$ Fernández $D G^{10}$

${ }^{1}$ Endocrinóloga, Hospital de San José. Profesora asociada, Fundación Universitaria de Ciencias de la Salud; Bogotá, Colombia.

${ }^{2}$ Endocrinólogo. Director de Osteollanos; Villavicencio, Colombia. ${ }^{3}$ Nutricionista, Colsanitas; Bogotá, Colombia.

${ }^{4}$ Médico internista, Hospital de San José. Fellow en Endocrinología, Fundación Universitaria de Ciencias de la Salud; Bogotá, Colombia.

${ }^{5}$ Educadora en diabetes y osteoporosis. Programa de fractura, Hospital de San José; Bogotá Colombia.

${ }^{6}$ Endocrinóloga, Hospital de San José; Bogotá, Colombia.

${ }^{7}$ Médico internista, Hospital San José Infantil. Fellow en

Endocrinología, Fundación Universitaria de Ciencias de la Salud; Bogotá, Colombia.

${ }^{8}$ Endocrinóloga, Colsanitas; Medellín, Colombia.

${ }^{9}$ Nutricionista. Profesora, Universidad de la Sabana; Bogotá, Colombia.

${ }^{10}$ Reumatólogo y epidemiólogo clínico, Hospital Universitario San Ignacio. Profesor asistente, Pontificia Universidad Javeriana; Bogotá, Colombia.

Autor de correspondencia: Adriana Medina, MD

Correo electrónico: adrimedor@hotmail.com

Fecha de recepción: 27/01/2020

Fecha de aceptación: 5/03/2020

\section{Resumen}

El calcio es el mineral más abundante del cuerpo y está contenido, en su mayoría, en el esqueleto. La ingesta de este elemento depende de la edad y de la condición del individuo.
En efecto, existe una alta deficiencia en el consumo de calcio en la dieta a nivel mundial y nacional, según los estudios recientes que motivaron a la Asociación Colombiana de Osteoporosis y Metabolismo Mineral (ACOMM) para la elaboración de este manuscrito.

En el desarrollo de este texto, se contactaron especialistas de diferentes disciplinas, con interés académico en salud ósea y nutrición. Mediante la consulta de literatura científica, de bases de datos como Embase, Pubmed, Scielo y Google Académico, y con la asesoría de un epidemiólogo, se profundizó en las propiedades de los lácteos, las características de la leche y sus derivados, sus beneficios para la salud ósea (densidad ósea y prevención de fracturas) y el riesgo cardiovascular.

Además, se registraron las recomendaciones del consumo lácteo para la población y se desmitificaron algunos conceptos que no tenían bases en la evidencia científica, los cuales han causado a una disminución en el consumo de calcio de la dieta, con un impacto negativo en la salud ósea.

Palabras clave: productos lácteos, leche, fracturas, dieta, osteoporosis, calcio.

\section{Abstract}

Calcium is the most abundant mineral in the body, mostly contained in the skeleton. Calcium intake depends on the age and patient condition. There is a high deficiency in dietary calcium intake worldwide and nationally, according to recent studies, which motivated Asociación Colombiana de Osteoporosis y Metabolismo Mineral (ACOMM) for the preparation of this 
manuscript, inviting specialists from different disciplines with academic interest in bone health and nutrition.

Through the consultation of scientific literature of databases such as Embase, Pubmed, Scielo and Google Scholar, and with the advice of an epidemiologist, it was studied thoroughly the properties of dairy products, the characteristics of dairy products, their benefits for bone health (bone density and fracture prevention), cardiovascular risk and finally, the dairy consumption recommendations for the population, demystifying some concepts without basis in scientific evidence, which have led to a decrease in the consumption of dietary calcium with a negative impact on bone health.

Keywords: dairy products, milk, fractures, diet, osteoporosis, calcium.

\section{Introducción}

El mineral más abundante en el cuerpo humano es el calcio, con múltiples funciones a nivel cardiovascular, muscular, neuronal, hormonal y óseo, entre otras. El $99 \%$ del calcio está contenido en el esqueleto, por lo que una baja ingesta se ha relacionado con el deterioro de la salud ósea en el adulto, pues su consumo adecuado es fundamental para lograr el pico de masa ósea en la juventud. La absorción de calcio proveniente de la dieta o suplementos es de apenas del $30 \%$, razón por la cual se debe optimizar su ingesta para garantizar un aporte adecuado, disminuyendo la prevalencia de osteoporosis, que genera riesgo de fractura y aumento de la mortalidad (1-3).

En ese contexto, la recomendación de ingesta de calcio adecuada depende de la edad y la condición del individuo. En la Tabla 1 se describen las recomendaciones de ingesta diaria.

Tabla 1. Recomendaciones dietarias de calcio por edad o condición

\begin{tabular}{l|c}
\hline Edad o condición de calcio (mg/d) & $\begin{array}{c}\text { Recomendación } \\
\text { diaria permitida }\end{array}$ \\
\hline Niños & $800-1000$ \\
\hline Adolescentes & 1300 \\
\hline Adultos (18-50 años) & 1000 \\
\hline $\begin{array}{l}\text { Mayores de } 50 \text { años y } \\
\text { posmenopáusicas }\end{array}$ & 1200 \\
\hline Embarazadas de 14-18 años & 1300 \\
\hline Embarazadas mayores de 18 años & 1000 \\
\hline
\end{tabular}

Modificado de las referencias 4-6.

El presente manuscrito pretende revisar la literatura disponible sobre las propiedades y características de los lácteos, su biodisponibilidad y su impacto en la densidad ósea, el riesgo de fracturas por fragilidad y el riesgo cardiovascular. La importancia de este trabajo radica en que la alta deficiencia en el consumo de lácteos a nivel mundial y nacional se basa, en algunas ocasiones, en información carente de soporte científico.

Así, para el desarrollo de este trabajo se contó con la participación de especialistas de diferentes disciplinas, con interés en la salud ósea y nutrición, convocados por la Asociación Colombiana de Osteoporosis y Metabolismo Mineral (ACOMM), que lideró todo el proceso. Se revisaron los ejes temáticos mencionados, mediante la búsqueda de literatura científica de bases de datos como Embase, Pubmed, Scielo y Google Académico, con la asesoría de un epidemiólogo, a fin de brindar las recomendaciones en el consumo de lácteos para la población adulta.

\section{Epidemiología del consumo de calcio a nivel mundial y en Colombia}

En un estudio de la International Osteoporosis Foundation (IOF), en el que se aplicó la calculadora de calcio $(6,7)$ a 6908 personas de 83 países, el consumo de calcio fue deficiente, según las recomendaciones de ingesta diaria del Instituto de Medicina (Institute of Medicine, IOM), en el $89 \%$ de los encuestados, con un promedio de consumo de calcio de 594 mg/d (6). En 2017, la IOF publicó una revisión sistemática de la ingesta de calcio global con los últimos datos mundiales disponibles de 74 países, de los 195 actuales, e incluyó en su mayoría, estudios no representativos de todo un país o basados en encuestas antiguas, determinando rangos mundiales de ingesta de calcio diaria que varían desde 175 a $1233 \mathrm{mg} /$ día. El país con el promedio más alto en aquel año fue Islandia con $1233 \mathrm{mg} /$ día, mientras que la nación con el promedio más bajo fue Nepal, con $175 \mathrm{mg} /$ día. En Estados Unidos la ingesta fue de $934 \mathrm{mg} /$ día, por encima de Canadá, con $787 \mathrm{mg} /$ día, y México, con $805 \mathrm{mg} /$ día. El promedio más bajo de ingesta fue para los países suramericanos, entre 400 y $700 \mathrm{mg} /$ día; en el primer lugar aparece Chile, con $702 \mathrm{mg} /$ día, seguido por Brasil, con 505 mg/día, Bolivia, con 458 mg/día, Argentina, con 427 mg/día, Ecuador, con $384 \mathrm{mg} /$ día, y Colombia, en el último lugar, con $297 \mathrm{mg} /$ día. Solo los países del norte de Europa tienen un promedio $>1000 \mathrm{mg} /$ día (2).

Recientemente, en Colombia hemos contado con dos estudios observacionales, descriptivos, de corte transversal. Uno fue realizado en 5 ciudades (Pereira, Medellín, Bogotá, Cali y Bucaramanga), mientras que el otro se desarrolló en Bogotå. El primero contó con 179 participantes y describió la ingesta de calcio promedio de $601 \mathrm{mg} /$ día; el grupo con menor consumo estaba entre los 30 y 34 años, con 553 mg/día y el de mayor consumo de 35 a 39 años, con $972 \mathrm{mg} /$ día (8). El segundo estudio fue desarrollado en Bogotá y tuvo 450 participantes, de quienes se obtuvo un valor promedio de ingesta de 724,5 mg/día, siendo mayor en el sexo masculino (731,5 mg/día), en 
comparación con el femenino (717,5 mg/día), así como más alto cuanto más elevado era el estrato social (9).

\section{Propiedades nutricionales de los lácteos}

La leche y sus derivados son alimentos que contienen nutrientes esenciales en las diferentes etapas de la vida. Por tanto, su ingesta se refleja de forma positiva en la salud y el bienestar de los seres humanos. El valor nutricional de los lácteos es variable de acuerdo con el tipo de producto que se consuma, pero, en general, estos contienen los siguientes componentes:

- Proteína: se encuentra en una cantidad cercana a 3 g/100 $\mathrm{mL}$ en la leche entera, mientras que en el yogur griego alcanza los $6 \mathrm{~g} / 100 \mathrm{~mL}$. Los lácteos contienen diferentes tipos de proteína, pero su mayor componente es la caseína, la cual facilita la absorción del calcio $(10,11)$.

- Carbohidratos: los lácteos contienen lactosa, la cual, según la Organización Mundial de la Salud (OMS), no genera efectos adversos al tratarse de un azúcar natural. En la leche entera, se encuentra una cantidad cercana a 4,7 g de lactosa/100 mL, y en los lácteos fermentados, como los yogures o los quesos, disminuye la concentración de lactosa o no está presente. Por tanto, las personas intolerantes a ella pueden consumir este tipo de productos $(10,11)$.

- Grasa: la cantidad de esta es variable de acuerdo con el tipo de lácteo, ya que se pueden encontrar enteros, descremados o semidescremados. La leche contiene ácidos grasos de cadena corta, que por su fácil absorción favorecen su digestibilidad. Además, son una fuente de energía con una menor tendencia a ser almacenados en el tejido adiposo. La leche contiene también ácidos grasos saturados e insaturados $(11,12)$.

- Minerales y vitaminas: la leche y sus derivados reúnen condiciones óptimas de asimilación, tales como una ligera acidez (todavía mayor en el yogur), la presencia de proteínas y un buen equilibrio con el aporte de fósforo. $\mathrm{Al}$ contrario de otras bebidas derivadas de vegetales, la leche presenta una alta calidad nutricional no solo determinada por su contenido alto de calcio, sino también de fósforo, magnesio, zinc, yodo y selenio, y de vitaminas como la $\mathrm{A}$, D y el complejo $B$, especialmente la vitamina $B_{12}(11,12)$.

- Agua: ayuda en la hidratación del organismo y su función consiste en distribuir los componentes del lácteo.

De otro lado, la leche se encuentra disponible en diferentes presentaciones:

- Entera: es a la que no se le ha realizado ningún tipo de reducción en el contenido de grasa y contiene entre el 3 y el $3,5 \%$ de grasa por cada $100 \mathrm{~mL}$

- Semidescremada: se ha disminuido el contenido de grasa (aproximadamente en un $50 \%$ ) y contiene entre el 1,5 y el $2 \%$ de grasa por cada $100 \mathrm{~mL}$.
- Descremada: tiene menos del 0,5\% de grasa por cada $100 \mathrm{~mL}$.

- Deslactosada: es a la que se le adiciona la enzima lactasa, con el fin de mejorar la digestibilidad en personas con intolerancia a la lactosa. Esta leche tiene un sabor dulce, debido al desdoblamiento de la lactosa mediante la lactasa adicionada y no a que contengan azúcar adicional.

- Deslactosada-descremada: es de mejor digestibilidad por ser deslactosada y tiene menos del 0,5 \% de grasa por cada $100 \mathrm{~mL}(13,14)$.

Con respecto a los quesos, su aporte nutricional está dado principalmente por proteína, grasa y calcio. Dicho valor nutricional puede variar de acuerdo con el tipo de leche utilizada (vaca, oveja, búfala) y con el proceso de elaboración y maduración, y se pueden clasificar por:

- Consistencia

- Quesos blandos (contienen hasta un $80 \%$ de agua): cottage, requesón, brie, camembert.

- Quesos duros (solo un 30 \% de agua): parmesano, gruyer, emmental, provolone.

- Contenido graso

- Queso doble crema: mínimo el $60 \%$ de grasa.

- Queso graso: 40-45\% de grasa.

- Queso semigraso: 25-40 \% de grasa.

- Queso magro: máximo del $25 \%$ de grasa.

- Grado de maduración

- Quesos curados.

- Quesos semicurados.

Los quesos duros contienen $240 \mathrm{mg}$ de calcio por cada $30 \mathrm{~g}$ de porción, mientras que los blandos cuentan con $240 \mathrm{mg}$ por cada 60 g de porción. Una porción de 60 g de queso mozzarella, que es semigraso, contiene $242 \mathrm{mg}$ de calcio, y el queso crema, $180 \mathrm{mg}$ de calcio por cada $30 \mathrm{~g}$ de porción (15).

Es importante aclarar que los lácteos en los cuales se realiza un proceso de descreme o una modificación en algún otro tipo de nutriente, aportan la misma cantidad de calcio que los enteros (12-14). En vista de las propiedades descritas de los lácteos, puede afirmarse que estos son útiles durante toda la vida, a fin de garantizar la presencia de un alimento completo y que es, por excelencia, fuente de calcio, lo que repercute en la conservación de la masa ósea y en la prevención de la osteoporosis (12-14).

Sin embargo, con el paso del tiempo, los patrones dietarios han variado y se han encontrado cambios en la tendencia de consumo de productos lácteos. Así, la popularidad de las bebidas azucaradas y jugos de frutas puede influir en la sustitución de la ingesta de leche. Según los datos del Continuing Survey of Food Intakes by Individuals (CSFII), entre 1994 y 1996, por cada $30 \mathrm{~mL}$ de reducción en consumo de leche en niños de 5-18 años, hay un aumento de $126 \mathrm{~mL}$ en la ingesta de bebidas 
azucaradas, con un incremento neto de $31 \mathrm{kcal}$ y una pérdida de calcio de $34 \mathrm{mg}$ por cada $30 \mathrm{~mL}$ de leche desplazada (16).

\section{Biodisponibilidad de los lácteos}

Los lácteos son alimentos complejos que aportan una gran variedad de nutrientes. Los derivados lácteos tienen un contenido nutricional diferente que está condicionado por el tipo y la composición de la leche empleada, así como por el proceso de elaboración al que haya sido sometida para obtener el producto final (17).

En los quesos, el contenido de minerales es mayor que en la leche, especialmente en los maduros, por su menor cantidad de agua. La biodisponibilidad de los minerales puede aumentarse con la elaboración de los lácteos, es así como en leches fermentadas, debido a la acidez del medio, algunos minerales como hierro, cobre y zinc pueden formar sales parcialmente solubles, y los mayoritarios como calcio, fósforo y magnesio, presentes en la fase coloidal, pasan a la fase soluble, lo que facilita su absorción. Asimismo, las enzimas microbianas hidrolizan la proteína láctea, lo cual contribuye a la formación de complejos entre péptidos, aminoácidos y iones como calcio, magnesio y fósforo, facilitando su absorción (17).

La biodisponibilidad del calcio está condicionada por otros factores que pueden aumentarla o disminuirla. En este sentido, los lácteos tienen características únicas que los diferencian de otros alimentos o de los suplementos, y que son importantes para garantizar la absorción de este mineral en condiciones fisiológicas desfavorables. Debido a que está unido a péptidos y proteínas, es más probable que el calcio de la leche se mantenga en solución cuando el pH es desfavorable, como la aclorhidria (17). Bajo condiciones de dieta normales, cerca del 30-40\% del calcio contenido en la leche y el queso es absorbido en el intestino, ya sea por transporte dependiente de la vitamina D a través del duodeno, difusión facilitada o influencia de la lactosa en el intestino delgado distal, por vía paracelular. Solo el 28-30\% del calcio contenido en los cereales se absorbe a partir de los cereales fortificados $(18,19)$.

Por otro lado, el calcio puede ser absorbido en ausencia de vitamina D gracias a la influencia de otros elementos como lactosa o lactoalbúmina. Los productos lácteos no contienen fitatos, oxalatos, ácidos urónicos o polifenoles presentes en los alimentos de origen vegetal y que dificultan la absorción del calcio. Además, el efecto hipercalciúrico de las proteínas lácteas se compensa por la acción hipocalciúrica del fósforo y potasio (17). Las fuentes vegetales de calcio pueden ser menos biodisponibles, aunque no se ha observado un mayor riesgo de fracturas en la población vegana. En un estudio que comparó la densidad mineral ósea (DMO) y los marcadores de salud ósea de omnívoros, ovolactovegetarianos y veganos sanos sin obesidad, se encontró que la DMO estaba reducida en los grupos de individuos que no consumían carne, en un porcentaje del 4-5 \% aunque sin significancia estadística, mientras que la excreción de calcio en 24 horas fue significativamente mayor en los consumidores de carne, en comparación con los vegetarianos (18). Aunque es posible cubrir las recomendaciones de calcio con una dieta exenta de lácteos, la mayoría de los estudios coinciden en afirmar que su eliminación total dificulta cubrir adecuadamente la ingesta recomendada en todos los grupos de edad y, al mismo tiempo, comprometer el aporte de otros nutrientes esenciales (17).

Así pues, los lácteos son una fuente fundamental de calcio y proporcionan del 55 al $70 \%$ del calcio dietario, así como el 20-28 \% de los requerimientos de proteína de alta calidad, con péptidos y factores bioactivos que tienen efecto en el crecimiento según la edad del consumidor. La porción lipídica de los productos lácteos suple energía y ácidos grasos esenciales y no esenciales $(17,19)$. Uno de los posibles beneficios del consumo de leche contra el riesgo de fractura puede estar asociado al aumento de las concentraciones sanguíneas del factor de crecimiento similar a la insulina (Insulin-like Growth Factor-1, IGF 1), relacionado con el alto contenido proteico de la leche, lo cual favorece la ganancia de masa muscular y ósea $(20,21)$.

\section{¿El consumo de lácteos mejora la DMO en los adultos?}

Existe controversia entre las diversas revisiones y los metaanálisis acerca del consumo de lácteos para aumentar la DMO y disminuir el riesgo de fractura; por esta razón, existe cierto grado de dificultad en la realización e interpretación de dichos estudios por varios motivos, entre ellos el número de participantes, la medición de desenlaces, la variación del consumo de calcio entre cada estudio, los diferentes puntos de corte de DMO, el uso concomitante de suplementos, la variabilidad genética de las poblaciones, la masa muscular, la fuerza y la actividad física, entre otros.

El mecanismo propuesto para que la ingesta de calcio mejore la salud ósea ocurre a través del aumento en la DMO, la cual es un indicador del riesgo de fractura. En un metaanalisis publicado en 2015, se observó que el aumento del consumo de calcio a partir de la dieta incrementó la DMO en un 0,7-1,8 \% luego de dos años a nivel de columna lumbar, cuello femoral, cadera total y cuerpo total, sin encontrar efecto en antebrazo ni una relación entre dosis-respuesta (22). Si extrapolamos estos resultados a la reduccion del riesgo de fractura, se estima que por cada 1-2 \% de ganancia de masa ósea, se reduce entre un 5-10\% el riesgo de fractura en los sitios analizados (22).

En un metanálisis publicado en 2018 que incluyó a 175060 participantes, se estudió el efecto de varios patrones dietarios sobre la DMO y las fracturas. Las dietas a base de lácteos, frutas, verduras, cereales, carnes magras y leguminosas se asociaron con mayor DMO y menor riesgo de fracturas en adolescentes y adultos mayores, por otra parte, en aquellos es- 
tudios en los que se consideró un patrón alimentario no saludable, caracterizado por carnes procesadas, gaseosas, granos refinados, comidas rápidas y azúcares, se observó una menor DMO entre los adultos mayores (23).

En un estudio que incluyó a 80 mujeres adolescentes de la secundaria de Sheffield (Inglaterra), con un promedio de ingesta del calcio de la leche de $1125 \mathrm{mg} /$ día, un grupo control con $746 \mathrm{mg} /$ día y una referencia de consumo de calcio para el Reino Unido de $800 \mathrm{mg} /$ día en niñas de esta de edad, se encontró una ganancia del 17,6 \% de masa ósea corporal total en el grupo de mayor ingesta de leche frente al $15,7 \%$ en el grupo control; este hallazgo se asoció al efecto del IGF-1 sobre la formación ósea, el cual es estimulado por los lácteos (24).

Por otro lado, un estudio de corte transversal, que incluyó a 2094 habitantes de 70 años de Umeå (Suecia), evaluó la tibia y el radio no dominantes mediante DXA y tomografía cuantitativa periférica (Peripheral Quantitative Computed Tomography, pQCT) y no se observó una asociación entre la cantidad de productos lácteos ingeridos y la DMO volumétrica trabecular y cortical, ni con el grosor cortical en la tibia y en el radio, concluyendo que existe una correlación positiva débil del consumo de lácteos con el área transversal trabecular y cortical en la tibia (25).

Para finalizar, una revisión sistemática reciente que tuvo en cuenta los metanálisis anteriores, encontró que el calcio proveniente de los productos lácteos, con y sin vitamina D, afecta positivamente la mineralización ósea en niños y adultos con una ingesta basal baja de calcio. Según los estudios que compararon el consumo de lácteos frente al de suplementos de calcio, no se halló mayor ganancia de DMO en este último, en relación con el primero (26).

\section{¿El consumo de lácteos reduce el riesgo de fracturas por fragilidad en los adultos?}

Los estudios sobre el consumo de calcio en la dieta, en cuanto a su efecto en la densidad mineral o marcadores de resorción óseos muestran resultados benéficos de este elemento sobre la salud de los huesos. Sin embargo, la relación entre la ingesta de lácteos y la disminución en el riesgo de fractura es aún controvertida y contradictoria (27).

Un estudio de revisión sistemática y metaanálisis elaborado en 2009, y actualizado en 2014, incluyó 53 trabajos que contaron con 91791 participantes. En ellos, se utilizó la vitamina D (con o sin calcio), calcitriol o alfacalcidol, y se encontró que la vitamina D sola no es efectiva en la prevención de una fractura de cadera (11 estudios: 27693 participantes; riesgo relativo [RR]: 1,12; intervalo de confianza [IC], $95 \%$ : 0,98-1,29) o de cualquier nueva fractura (15 estudios: 28271 participantes; RR: 1,03; IC, 95 \%: 0,96-1,11). Dentro de este contexto, la vitamina D con calcio mostró generar una pequeña disminución del riesgo de fractura de cadera, así como una reducción significativa en la incidencia de nuevas fracturas no vertebrales. Esto sugiere que aquellos con un mejor índice de ambos nutrientes tienden a contar con un menor riesgo de fracturas (9 estudios: 49853 participantes; RR: 0,84; IC, 95 \%: 0,74-0,96). En este sentido, el consumo de lácteos mejora el aporte de calcio y favorece la ingesta de vitamina $\mathrm{D}$, dado que muchos de estos productos son fortificados (28).

Un metaanálisis publicado en 2007, en el que se incluyeron 29 ensayos clínicos controlados aleatorizados, con una muestra de 63897 individuos mayores de 50 años, concluyó que la suplementación con $1200 \mathrm{mg}$ de calcio, en conjunto con 800 UI de vitamina D al día, previno las fracturas y disminuyó la pérdida de masa ósea en este subgrupo de población. Esto indica que existe un efecto sinérgico entre ambos nutrientes presentes en los productos lácteos, con una disminución del riesgo de fractura del $24 \%(p<0,0001)$. También se encontró un mayor efecto en individuos con baja ingesta basal de calcio $(<700 \mathrm{mg} / \mathrm{d})$, con un RR de 0,80 frente a 0,95 ( $p=0,008$ ) (29).

Entre tanto, una revisión sistemática y un metaanálisis de estudios prospectivos de cohortes evaluaron la relación entre el consumo de leche y el riesgo de fracturas. Además, se valoró la aparición de fracturas de cadera como desenlace principal en hombres y mujeres de 47 a 71 años, para lo cual la media de seguimiento varió entre 3 y 26 años, en poblaciones de Estados Unidos, Escandinavia y Japón. También se realizó la evaluación de cohortes de otras regiones del mundo (Europa, Australia y Canadá). Estos estudios contaron con 270251 participantes que sumaron 3769 fracturas. En ellos, no se encontró una asociación entre el consumo de leche y la fractura de cadera. Sin embargo, cuando se excluyó uno de los estudios suecos y se disminuyó la heterogeneidad de los trabajos incluidos, se observó una disminución del riesgo significativo de $5 \%$ para fractura de cadera por vaso de leche diario. Asimismo, los resultados mostraron que en la población de mujeres (6 estudios: 195102 mujeres y 3574 fracturas de cadera) no hubo una asociación general entre la ingesta total de leche y el riesgo de fractura de cadera (RR agrupado por vaso de leche por día: 0,99; IC, 95 \%: 0,96-1,02; prueba Q, $p=$ 37) y en los hombres (3 estudios: 75149 participantes y 195 fracturas de cadera), el RR agrupado por vaso de leche diario fue de 0,91 (IC, $95 \%$ : 0,81-1,01). No hubo entonces una asociación general entre la ingesta de leche y el riesgo de fractura de cadera en las mujeres, pero en los hombres es necesario obtener más datos (30).

Por otro lado, el metaanálisis de Kanis y colaboradores, que incluyó 6 cohortes prospectivas en 2005, no halló una relación significativa entre el bajo consumo de leche y el riesgo de fractura de cadera. No obstante, el elemento de recolección sobre la ingesta baja de leche tuvo poco o ningún valor en las estrategias de detección de casos (31). En contraste, el metaanálisis de Bian y colaboradores, que incluyó 10 estudios de cohortes y 8 estudios de casos y controles, tuvo resultados 
discordantes. Por un lado, en los estudios de casos y controles se observó que el consumo de leche se asoció con una disminución promedio del $29 \%$ en el riesgo de fractura de cadera; y, por otra parte, en los estudios de cohorte se encontró que no existe evidencia consistente sobre la relación entre la ingesta de leche y el riesgo de fractura de cadera (32).

Dos grandes cohortes de Estados Unidos, el estudio de salud de enfermeras (Nurses Health Study, NHS) en mujeres (con un tiempo medio de seguimiento de 20,8 años) y el estudio de seguimiento de profesionales de la salud (Health Professionals Follow-up Study, HPFS) en hombres (con un tiempo medio de seguimiento de 17,5 años) examinaron el consumo a largo plazo de leche y de otros productos lácteos, en relación con el riesgo de fractura de cadera. Estos trabajos hallaron que una elevación en el consumo de leche en adultos mayores se asoció con un menor riesgo de fractura de cadera de $8 \%$ por cada porción de leche por día (RR: 0,92; IC, 95 \%: 0,87-0,97) (33).

Según la cohorte de Framingham, con 764 adultos con una edad promedio de 77 años, una mayor ingesta de leche (más de 1 vaso de leche por día) y leche más yogur disminuyó el riesgo de fractura de cadera en un 40 \% en comparación con la ausencia de ingesta (34).

En 1997, un metaanálisis que incluyó 16 estudios concluyó que una ingesta de calcio de $1000 \mathrm{mg} /$ día a partir de suplementos o dieta reducía el riesgo de fractura en un 34 y un $24 \%$, respectivamente (odds ratio [OR]: 0,96; IC, $95 \%$ : 0,93-0,99) (35).

Estudios desarrollados en el sur de Europa observaron un mayor riesgo de fracturas en personas con bajo consumo de leche y queso (baja ingesta de calcio), pero no una reducción del riesgo en aquellas con una ingesta por encima del promedio. Así, en poblaciones con alto riesgo de fractura osteoporótica existe un umbral de incremento de riesgo de fractura que oscila entre 400 y $500 \mathrm{mg} / \mathrm{d}$ de ingesta de calcio. Sin embargo, no hay un beneficio adicional asociado con un consumo por encima de lo recomendado (36).

Una posible explicación sobre las contradicciones entre los estudios puede ser la presencia de diversos factores de confusión y el riesgo de sesgos. Estos aspectos son difíciles de controlar, ya que no hay una sola herramienta de recolección de datos y muchos investigadores requieren de autoinformes que podrían no estimar realmente el consumo de calcio en cada individuo, lo que contribuye a la alta heterogeneidad en los resultados. Además, es importante considerar la ingesta de leches fortificadas que pueden afectar la salud ósea, como lo demuestra el estudio de Kruger en el que la leche fortificada disminuyó los marcadores de recambio óseo e incrementó la DMO en el cuello femoral (37). En los diferentes estudios se presentan sesgos como: la ingesta previa de calcio, la menopausia temprana, el tabaquismo, los malos hábitos alimentarios y el sedentarismo. En suma, en estos trabajos no se ha comprobado que el consumo de lácteos aumente el riesgo de fracturas por fragilidad y, por el contrario, sí podría prevenirlas (27).

\section{¿Existe riesgo cardiovascular con el consumo de lácteos?}

Una gran controversia se suscita a nivel mundial al evaluar el papel de la dieta, los hábitos de vida y su contribución en el proceso salud-enfermedad. Numerosos estudios y metaanálisis han evaluado qué compuestos de la dieta generan un impacto positivo o negativo en el riesgo de presentar desenlaces cardiovasculares adversos, partiendo de la premisa epidemiológica que las enfermedades cardiovasculares son la principal causa de muerte e incapacidad a nivel mundial. Por supuesto, la leche y sus derivados no han escapado a dichos escrutinios, y como fuente alimenticia de gran parte de la población ha sido objeto de debate al respecto. A fin de poner en contexto la discusión sobre la leche y el riesgo cardiovascular, se revisó la composición nutricional de este alimento, encontrando que el $20 \%$ del contenido proteico de la leche corresponde a las proteínas del suero, las cuales están integradas por aminoácidos de cadena ramificada (leucina, isoleucina y valina), mientras que las proteínas insolubles — que representan el $80 \%$ están constituidas por las caseínas, que contienen histidina, metionina y fenilalanina. Estos aminoácidos son de alto valor biológico y tienen propiedades antimicrobianas, antihipertensivas, antitrombóticas e inmunomoduladoras (38). En esa medida, las propiedades antihipertensivas de la leche y sus derivados se asocian a péptidos procedentes de la caseína, los cuales inhiben la enzima convertidora de angiotensina y pueden obtenerse de la leche agria y el queso suizo $(38,39)$. Con respecto a las propiedades antitrombóticas, ciertos péptidos derivados de la hidrólisis enzimática de la caseína tienen similitud estructural con la cadena del fibrinógeno humano. Entre estos péptidos se halla la casoplatelina, que inhibe la agregación plaquetaria inducida por el adenosín difosfato (adenosine diphosphate, ADP) y la unión al fibrinógeno (40).

De otro lado, el contenido graso de la leche es una de las cuestiones que despierta especial interés en el debate alrededor de su papel en el riesgo cardiovascular. Así, la leche tiene una alta cantidad de grasas saturadas (70 \%) que se han asociado a un mayor riesgo de enfermedades cardiovasculares (41) y sus efectos parecen mediados, principalmente, por el aumento en las concentraciones y mayor oxidación de las lipoproteínas de baja densidad (Low Density Lipoproteins, LDL). En contraposición, se ha demostrado que los ácidos láurico y esteárico (este último es un componente de los ácidos grasos saturados de la leche) disminuyen los niveles de colesterol total y aumentan las lipoproteínas de alta densidad (High Density Lipoproteins, HDL), lo que genera una acción protectora a nivel cardiovascular $(40,42,43)$. Además de ello, los ácidos grasos insaturados y polinsaturados, tales como el oleico, el linoleico y $\alpha$-linolénico, han demostrado tener propiedades antiarrítmicas, antiinflamatorias, antitrombóticas e hipolipemiantes, documentadas en estudios clínicos como el GISSI 
Prevenzione (43). Esto es importante de considerar, dado que los lácteos y sus derivados aportan el 70 \% del ácido linoleico ingerido a diario.

Así pues, para zanjar la discusión sobre el contenido graso en la dieta y el riesgo cardiovascular, es fundamental mencionar el estudio PURE (Prospective Urban Rural Epidemiology), que es una cohorte con 135335 individuos de 18 países, entre los 35-70 años, con una mediana de seguimiento de 7,4 años, con un registro de ingesta diario de alimentos mediante cuestionarios validados de alimentación. Durante el seguimiento, se reportaron 5796 muertes, así como 4784 eventos de enfermedades cardiovasculares mayores, y se determinó que la ingesta de grasas se asoció con un menor riesgo de mortalidad general, $24 \%$ para grasas saturadas (principal componente de la leche) y $21 \%$ menor riesgo de muerte por causa de accidentes cerebrovasculares, sin documentarse un incremento del riesgo de infarto agudo de miocardio o de muerte por enfermedad cardiovascular asociado (44).

Asimismo, el estudio de Rotterdam examinó la relación existente entre la ingesta de lácteos y la hipertensión incidental. Dicho trabajo se realizó en 2245 participantes mayores de 55 años, con datos de la dieta y la presión arterial, y con un seguimiento de 2 años. Como resultado, se reportó una reducción del 20 \% en la incidencia de la hipertensión asociada con el consumo de lácteos bajos en grasa (45).

También hay datos controvertidos alrededor de la asociación del consumo de leche y el riesgo cardiovascular. En primer lugar, se halló el estudio ATBC (Alpha-Tocopherol, Beta-Carotene Cancer Prevention), realizado por Larsson y colaboradores (46). Dicho trabajo documentó una relación positiva entre la ingesta de leche entera y el riesgo de hemorragia intracerebral.

En contraposición, existen metanálisis y revisiones sistemáticas que han documentado una asociación inversa débil entre la ingesta de leche y el riesgo de enfermedades cardiovasculares, sin que se devele una relación significativa entre su consumo y el riesgo de accidente cerebrovascular o enfermedad coronaria (47). Estos hallazgos han sido respaldados en grandes cohortes como la de Caerphilly (Gales), en la que se reportó una discreta disminución del riesgo cardiovascular (infarto agudo de miocardio y accidente cerebrovascular isquémico) (48).

\section{Intolerancia a la lactosa}

La intolerancia a la lactosa se refiere a los síntomas resultantes de su ingestión como flatos, distensión abdominal y diarrea, los cuales pueden producirse por causas genéticas o adquiridas, así como por infecciones intestinales, enfermedades inflamatorias intestinales o cirugías del intestino delgado. En este contexto, las personas con lactasa no persistente son aquellas con declinación natural en la actividad de la lactasa a menos de $10 \mu \mathrm{g}$ de tejido, lo cual produce una habilidad míni- ma en los adultos para digerir la lactosa (49). Entre tanto, en los niños menores de 4 años, la deficiencia de lactosa es casi nula; por tal razón, es importante aprovechar esta ventana de tiempo para lograr el mayor consumo y el depósito de calcio en los huesos (50). Después de esta edad, la decisión de retirar los lácteos de la dieta ya sea porque se presentan síntomas de intolerancia a la lactosa, por recomendación médica o por elección personal, dificulta a las personas alcanzar el aporte de calcio y de proteína necesario.

Una opción válida en estos casos es incluir alimentos lácteos con un bajo contenido de lactosa o evitar el consumo de dosis elevadas de lactosa en una sola toma debido a que ingerir porciones más pequeñas, repartidas a lo largo del día, no produce síntomas gastrointestinales en la mayoría de las personas, lo que permite un mejor manejo desde el punto de vista nutricional (51). Aunque en el mercado existen productos a base de almendras o soya que intentan simular la composición de los productos lácteos, el aporte de calcio y proteína de estas bebidas (mal llamadas leches) es inferior al de los lácteos en 3 -10 veces $(7,51)$.

Por otra parte, la leche deslactosada que se encuentra en el mercado ha sido elaborada mediante un proceso en el cual el líquido pasa a través de un reactor que contiene a-galactosidasas microbianas. La enzima en el reactor digiere la lactosa en sus componentes monosacáridos: la glucosa y la galactosa. La primera tiene un poder edulcorante más alto que la lactosa original, lo cual permite que estos productos sean más dulces. El proceso de digestión de la lactosa no es totalmente eficiente, debido a que la actividad de las a-galactosidasas microbianas se inhibe por la acumulación de glucosa o galactosa. Este proceso de inhibición competitiva, causada por los productos de la reacción, es común en la mayoría de las enzimas y constituye un mecanismo de control natural de la actividad enzimática. Por tanto, el grado de hidrólisis de una gran parte de las leches deslactosadas disponibles en el mercado es del 70-85 \%, razón por la cual, en realidad, contienen menos lactosa que la leche original (51). En la Tabla 2 pueden observarse productos deslactosados y algunos derivados lácteos que contienen una menor cantidad de lactosa (49).

La adaptación colónica dada por la microbiota intestinal, a partir de los efectos prebióticos o probióticos, permite que aquellos individuos con lactasa no persistente consuman una mayor cantidad de productos lácteos (52). Los probióticos son microorganismos vivos que, al ser consumidos en las cantidades adecuadas, aportan resultados benéficos sobre la salud. Entre tanto, los prebióticos son carbohidratos mal digeridos, los cuales, al llegar al colon, son selectivamente metabolizados mediante la fermentación por cepas lácticas específicas, por lo que se producen bacterias (bifidobacterias y lactobacilos) que ejercen efectos digestivos positivos (52).

La microbiota del adulto es relativamente estable. No obstante, algunos factores como el consumo de antibióticos, 
ciertas enfermedades y la ingesta de dietas con alimentos muy refinados pueden alterar la flora intestinal. En el caso de las personas con intolerancia a la lactosa, se ha estudiado la relación entre el consumo de probióticos, presentes principalmente en el yogur, y la disminución de los síntomas. Asimismo, estudios in vitro han encontrado que el consumo de lactosa se asocia con el aumento de lactobacilos y bifidobacterias, lo cual determina el papel de la leche como prebiótico.

Tabla 2. Contenido de lactosa y calcio en algunos productos lácteos

\begin{tabular}{l|c|c}
\hline Producto lácteo/porción & $\begin{array}{c}\text { Contenido de } \\
\text { lactosa }(\mathbf{g})\end{array}$ & $\begin{array}{c}\text { Contenido de } \\
\text { calcio }(\mathbf{m g})\end{array}$ \\
\hline Leche entera: 1 taza & 12,8 & 276 \\
\hline $\begin{array}{l}\text { Yogur entero o descre- } \\
\text { mado: } 1 \text { taza }\end{array}$ & 8,4 & 448 \\
\hline Leche descremada: 1 taza & 12,2 & 285 \\
\hline Helado de vainilla: 1 taza & 4,9 & 92 \\
\hline Queso cheddar: $30 \mathrm{~g}$ & 0,07 & 224 \\
\hline Queso suizo: $30 \mathrm{~g}$ & 0,02 & 224 \\
\hline Queso cottage: 1 taza & 1,4 & 135 \\
\hline
\end{tabular}

Modificado de la referencia 40.

\section{Recomendaciones}

Los productos lácteos representan una buena fuente de calcio, dada su concentración, su alta tasa de absorción y su bajo costo relativo. Además, los lácteos proveen más proteína, calcio, magnesio, potasio, zinc y fósforo por caloría que cualquier otro alimento. Por tanto, el consumo de 3 a 4 porciones de lácteos por día permitiría alcanzar la recomendación de ingesta diaria de calcio.

Así, la leche y sus derivados pueden considerarse como componentes de una dieta equilibrada, ya que los nutrientes aportados por estos (grasas, calcio, proteínas, vitaminas y oli- goelementos) contribuyen a suplir otros alimentos que el organismo necesita, lo cual constituye un impacto positivo para la salud ósea.

Ante la presencia de intolerancia a la lactosa, la educación nutricional es clave para evitar el abandono definitivo de los lácteos. En este punto, pueden considerarse otras opciones válidas y efectivas a fin de minimizar el impacto de la lactosa no persistente; entre estas, la leche deslactosada, el fraccionamiento de los alimentos lácteos durante el día y el consumo de lácteos bajos en lactosa, pero ricos en probióticos, como los yogures.

Hasta el momento, con la evidencia disponible, la recomendación de incluir lácteos como parte de una alimentación balanceada ha mostrado beneficio en todas las etapas de la vida, ya que su ingesta aumenta la DMO, especialmente en los niños durante la etapa de ganancia de masa ósea. Además, los lácteos disminuyen el riesgo de fracturas por osteoporosis en los adultos mayores de 50 años y en las mujeres posmenopáusicas.

En consecuencia, incrementar la ingesta de calcio dietario parece ser más beneficioso en poblaciones con bajo consumo basal de este mineral. Las personas que ingieren lácteos como parte de su alimentación habitual han mostrado tener patrones nutricionales más saludables, los cuales podrían favorecer una buena salud ósea.

En Colombia, los últimos datos estadísticos evidencian un déficit de ingesta nutricional de calcio. Por tanto, se deben implementar estrategias desde las sociedades médicas científicas y el gobierno nacional que apunten a la ejecución de políticas de salud pública encaminadas a mejorar el consumo de este mineral en nuestra población.

\section{Declaración de fuentes de financiación y posibles conflictos de interés}

El presente documento se desarrolló con recursos provenientes del fondo de investigación de la Asociación Colombiana de Osteoporosis y Metabolismo Mineral (ACOMM). Los autores de este manuscrito no recibieron honorarios de ACOMM, ni de alguna otra entidad, por su participación en él. Tampoco declaran conflicto de interés, excepto la licenciada María Clara Obregón, quien ha prestado asesorías y consultorías para Alpina.

\section{Referencias}

1. Pu F, Chen N, Xue S. Calcium intake, calcium homeostasis and health. Food Science and Human Wellness. 2016;5(1):8-16.

2. Balk EM, Adam GP, Langberg VN, Earley A, Clark P, Ebeling PR, et al. Global dietary calcium intake among adults: a systematic review. Osteoporos Int. 2017;28(12):3315-3324

3. Beto JA. The role of calcium in human aging. Clin Nutr Res. 2015;4(1):1-8.

4. Bauer DC. Calcium Supplements and Fracture Prevention. N Engl J Med. 2013;369:1537-1543.

5. World Institute of Medicine. Dietary reference intakes for calcium and vitamin D. Washington, DC: National Academies Press, 2011.

6. World Osteoporosis Day [internet]. Survey results infographic [actualizada el 19 de octubre de 2015]. Disponible en: http://worldosteoporosisday.org/about-osteoporosis.

7. International Osteoporosis Foundation [internet]. Calcium calculator [actualizada el 19 de octubre de 2015]. Disponible en: https://www.iofbonehealth.org/calcium-calculator

8. Arenas H, Arias D, López V. Estamos en deuda con el calcio: determinación de consumo diario de calcio en Colombia. En: XV Congreso Colombiano de Endocrinología, Diabetes y Metabolismo. Pereira, Colombia; 2019

9. Coy A, Medina A, Rivera A, Sánchez P. Calcium intake in Colombia: are we still in deficit? Arch Osteoporos. 2020;15(71):3-6.

10. Instituto Colombiano del Bienestar Familiar (ICBF). Tabla de composición de alimentos colombianos 2015. Bogotá: ICBF; 2015.

11. Instituto Colombiano del Bienestar Familiar (ICBF). Encuesta Nacional de la Situación Nutricional en Colombia 2010 ENSIN: Resumen ejecutivo ENSIN 2010. Bogotá: ICBF; 2010. 
12. Sánchez MA, Murray RS, Montero J, Marchini M, Iglesias R, Saad G. Importancia de la leche y sus potenciales efectos en la salud humana/Revisión. Actualización en Nutrición. 2020;21(2):50-64.

13. Natursan [internet]. Tipos y variedades de leche [actualizada el $30 \mathrm{de}$ agosto de 2018]. Disponible en: https://www.natursan.net/tipos-y-variedades-de-leche/

14. Plaza J, Valero T, Varela G y Gil A. Fundación Española de la Nutrición (FEN); Fundación Iberoamericana de Nutrición (FINUT). La leche como vehículo de salud para la población: calcio y sus determinantes en la salud de la población española. Madrid: Editorial FEN y FINUT; 2017. p. 1-59.

15. Ross AC, Manson JE, Abrams SA, Aloia JF, Brannon PM, Clinton SK, et al. The 2011 report on dietary reference intakes for calcium and vitamin D from the Institute of Medicine: what clinicians need to know. J Clin Endocrinol Metab. 2011;96(1):53-8.

16. Dror DK, Allen LH. Dairy product intake in children and adolescents in developed countries: trends, nutritional contribution, and a review of association with health outcomes. Nutr Rev. 2014;72(2):68-81.

17. Moreno LA, Cervera P, Ortega RM, Díaz JJ, Baladia E, Basulto J, et al. Evidencia científica sobre el papel del yogur y otras leches fermentadas en la alimentación saludable de la población española. Nutr Hosp. 2013;28(6):2039-2089.

18. Knurick JR, Johnston CS, Wherry SJ, Aguayo I. Comparison of correlates of bone mineral density in individuals adhering to lacto-ovo, vegan, or omnivore diets: a cross-sectional investigation. Nutrients. 2015;7(5):34163426.

19. Rozenberg $\mathrm{S}$, Body JJ, Bruyère $\mathrm{O}$, Bergmann $\mathrm{P}$, Brandi $\mathrm{ML}$, Cooper $\mathrm{C}$, et al. Effects of Dairy Products Consumption on Health: Benefits and Beliefs--A Commentary from the Belgian Bone Club and the European Society for Clinical and Economic Aspects of Osteoporosis, Osteoarthritis and Musculoskeletal Diseases. Calcif Tissue Int. 2016;98(1):1-17.

20. Rizzoli R. Dairy products, yogurts, and bone health. Am J Clin Nutr. 2014;99(5 Suppl):1256S-62S.

21. Rizzoli R, Biver E. Effects of Fermented Milk Products on Bone. Calcif Tissue Int. 2018;102(4):489-500.

22. Tai V, Leung W, Grey A, Reid IR, Bolland MJ. Calcium intake and bone mineral density: systematic review and meta-analysis. BMJ. 2015;351:h4183.

23. Denova-Gutiérrez E, Méndez-Sánchez L, Muñoz-Aguirre P, Tucker KL, Clark P. Dietary Patterns, Bone Mineral Density, and Risk of Fractures: A Systematic Review and Meta-Analysis. Nutrients. 2018;10(12):1922.

24. Cadogan J, Eastell R, Jones N, Barker ME. Milk intake and bone mineral acquisition in adolescent girls: randomised, controlled intervention trial. BMJ. 1997;315(7118):1255-1260.

25. Hallkvist OM, Johansson J, Nordström A, Nordström P, Hult A. Dairy product intake and bone properties in 70-year-old men and women. Arch Osteoporos. 2018;13(1):9.

26. van den Heuvel EGHM, Steijns JMJM. Dairy products and bone health: how strong is the scientific evidence? Nutr Res Rev. 2018:31(2):164-178.

27. Fardellone $\mathrm{P}$. The effect of milk consumption on bone and fracture incidence, an update. Aging Clin Exp Res. 2019;31(6):759-764.

28. Avenell A, Mak JC, O'Connell D. Vitamin D and vitamin D analogues for preventing fractures in post-menopausal women and older men. Cochrane Database Syst Rev. 2014;2014(4):CD000227.

29. Tang BM, Eslick GD, Nowson C, Smith C, Bensoussan A. Use of calcium or calcium in combination with vitamin D supplementation to prevent fractures and bone loss in people aged 50 years and older: a meta-analysis. 2007. The Lancet. 2007;370(9588):657-666.

30. Bischoff-Ferrari HA, Dawson-Hughes B, Baron JA, Kanis JA, Orav EJ, Staehelin HB, et al. Milk intake and risk of hip fracture in men and women: a meta-analysis of prospective cohort studies. J Bone Miner Res. 2011;26(4):833-9.

31. Kanis JA, Johansson H, Oden A, De Laet C, Johnell O, Eisman JA, et al. A meta-analysis of milk intake and fracture risk: low utility for case finding. Osteoporos Int. 2005;16(7):799-804.
32. Bian S, Hu J, Zhang K, Wang Y, Yu M, Ma J. Dairy product consumption and risk of hip fracture: a systematic review and meta-analysis. BMC Public Health. 2018;18(1):165.

33. Feskanich D, Meyer HE, Fung TT, Bischoff-Ferrari HA, Willett WC. Milk and other dairy foods and risk of hip fracture in men and women. Osteoporos Int. 2018;29(2):385-396.

34. Sahni S, Mangano KM, Tucker KL, Kiel DP, Casey VA, Hannan MT. Protective association of milk intake on the risk of hip fracture: results from the Framingham Original Cohort. J Bone Miner Res. 2014;29(8):1756-1762.

35. Cumming RG, Nevitt MC. Calcium for prevention of osteoporotic fractures in postmenopausal women. J Bone Miner Res. 1997;12(9):1321-1329.

36. Prentice $\mathrm{A}$. What are the dietary requirements for calcium and vitamin $\mathrm{D}$ ? Calcif Tissue Int. 2002;70(2):83-88.

37. Michaëlsson K, Wolk A, Langenskiöld S, Basu S, Warensjö Lemming E, Melhus $\mathrm{H}$, et al. Milk intake and risk of mortality and fractures in women and men: cohort studies. BMJ. 2014;349:g6015.

38. Pereira PC. Milk nutritional composition and its role in human health. Nutrition. 2014;30(6):619-627.

39. Mills S, Ross RP, Hill C, Fitzgerald GF, Stanton C. Milk intelligence: Mining milk for bioactive substances associated with human health. International Dairy J. 2011;21(6):377-401.

40. Jollès $\mathrm{P}$, Lèvy-Toledano $\mathrm{S}$, Fiat $\mathrm{AM}$, Soria $\mathrm{C}$, Gillessen $\mathrm{D}$, Thomaidis $\mathrm{A}$, et al Analogy between fibrinogen and casein: Effect of an undecapeptide isolated from rc-casein on platelet function. 1986;158(2):379-382.

41. LaRosa JC, Hunninghake D, Bush D, Criqui MH, Getz GS, Gotto AM, et al. The cholesterol facts. A summary of the evidence relating dietary fats, serum cholesterol, and coronary heart disease. A joint statement by the American Heart Association and the National Heart, Lung, and Blood Institute. The Task Force on Cholesterol Issues, American Heart Association. Circulation. 1990;81(5):1721-33.

42. Fedačko J, Pella D, Mechírová V, Horvath P, Rybár RP, Varjassyová P, Vargová V. $\omega$-3 PUFAs-From dietary supplements to medicines. Pathophysiol. 2007;14(2):127-132.

43. Dietary supplementation with $n-3$ polyunsaturated fatty acids and vitamin E after myocardial infarction: results of the GISSI-Prevenzione trial. Gruppo Italiano per lo Studio della Sopravvivenza nell'Infarto miocardico. Lancet. 1999;354(9177):447-455.

44. Dehghan M, Mente A, Zhang X, Swaminathan S, Li W, Mohan V, et al. Associations of fats and carbohydrate intake with cardiovascular disease and mortality in 18 countries from five continents (PURE): a prospective cohort study. Lancet. 2017;390(10107):2050-2062.

45. Engberink MF, Hendriksen MA, Schouten EG, van Rooij FJ, Hofman A, Witteman JC, et al. Inverse association between dairy intake and hypertension: the Rotterdam Study. Am J Clin Nutr. 2009;89(6):1877-83.

46. Larsson SC, Männistö S, Virtanen MJ, Kontto J, Albanes D, Virtamo J. Dairy foods and risk of stroke. Epidemiology. 2009;20(3):355-360.

47. Soedamah-Muthu SS, Ding EL, Al-Delaimy WK, Hu FB, Engberink MF, Willett WC, et al. Milk and dairy consumption and incidence of cardiovascular diseases and all-cause mortality: dose-response meta-analysis of prospective cohort studies. Am J Clin Nutr. 2011;93(1):158-71.

48. Elwood PC, Strain JJ, Robson PJ, Fehily AM, Hughes J, Pickering J, et al. Milk consumption, stroke, and heart attack risk: evidence from the Caerphilly cohort of older men. J Epidemiol Community Health. 2005;59(6):502-5.

49. Lukito W, Malik SG, Surono IS, Wahlqvist ML. From 'lactose intolerance' to 'lactose nutrition'. Asia Pac J Clin Nutr. 2015;24 Suppl 1:S1-S8.

50. Torregrosa D, Mendoza E, Varela L, Villarreal J. Bases conceptuales del diagnóstico de intolerancia a lactosa, hipolactasia y mala digestión de lactosa. Salud Uninorte. 2015;31(1):101-117.

51. Rosado JL. Intolerancia a la lactosa. Gac Med Mex. 2016;152 (Suppl-1):67-73.

52. Szilagyi A. Adaptation to Lactose in Lactase Non Persistent People: Effects on Intolerance and the Relationship between Dairy Food Consumption and Evalution of Diseases. Nutrients. 2015;7(8):6751-6779. 


\section{(D) Hay órganos que sufren y no se ven ${ }^{1}$ La diabetes es más que glucosa ${ }^{1}$}

\section{Uso Temprano}
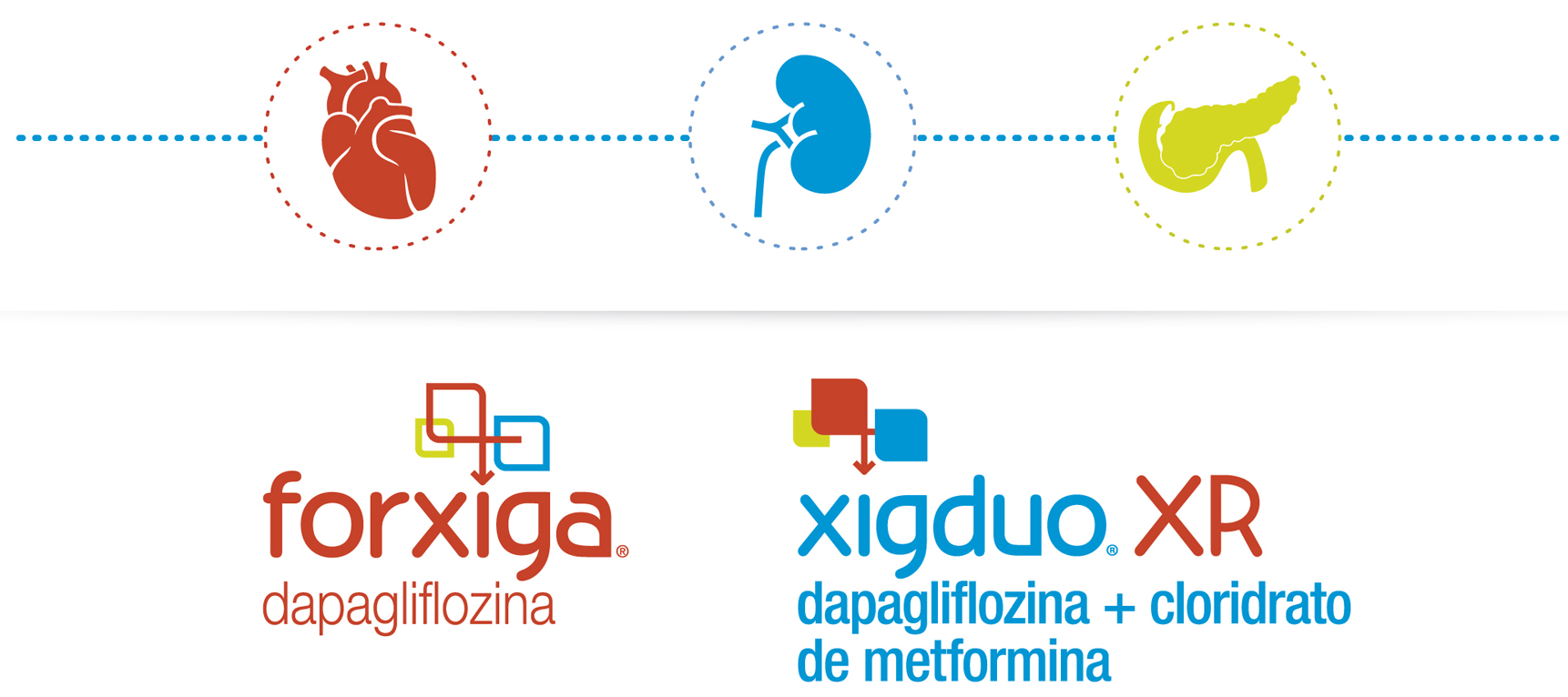

\section{EFICACIA: $\downarrow H b A 1 c$, Peso y PA².8}

\section{AMPLIO PERFIL DE SEGURIDAD2.3}

\section{CONCIENCIA CARDIORRENAL2-3,9}

Esto puede ocurrir de forma temprana, incluso antes de que su paciente sea dignosticado ${ }^{10}$ ¿Está su paciente con DMT2 protegido?

HbA1c: hemoglobina glucosilada; PA: Presión Arterial; DMT2: diabetes mellitus tipo 2.

Referencias: 1. Chen-Ku CH, et al. Vascular complications in patients with type 2 diabetes: prevalence and comorbidities in 6 countries of Latin America (a cohort
of the DISCOVER Study Program). Endocr Pract. 2019 Oct:25(10):994-1002. 2. IPP Forxiga. 3. IPP Xigduo XR. 4. Bailey, et al. Effect of dapagliflozin in patients with of the DISCOVER Study Program). Endocr Pract. 2019 Oct;25(10):994-1002. 2. IPP Forxiga. 3. IPP Xigduo XR. 4. Bailey, et al. Effect of dapagliflozin in patients with 5. Ferrannini E, et al. Diabetes Care 2010;33:2217-24. 6. Bolinder J. Diabetes Obes Metab. 2014 Feb;16(2):159-69. 7. Rosenstock, et al. Composite Endpoint Analysis of Dapagliflozin versus Saxagliptin as Add-On Therapy in Patients with Type 2 Diabetes Inadequately Controlled with Metformin. Presented at the 14th different risk categories: a meta-analysis. Cardiovasc Diabetol (2016) 15:37. 10. Gedebjerg, et al. Prevalence of micro- and macrovascular diabetes complications Jan;32(1):34-40. 